\title{
Roundabouts Improve the Safety of Intersections
}

\author{
Runfen Liu \\ (School of Transportation, Shijiazhuang Tiedao Univercity, Shijiazhuang 050043, Hebei, China)
}

\begin{abstract}
Roundabouts are generally safer than other forms of intersection in terms of aggregate crash statistics for low and medium traffic capacity conditions. This paper compared to values results injury crash rates roundabouts in different countries, Findings of these studies show a reduction in crashes by eliminating or altering conflict types after building a roundabout. at last, author offer optimum roundabout safety design notes.
\end{abstract}

Key words: roundabout; safety; crash

\section{Introduction}

Roundabouts may improve the safety of intersections by eliminating or altering conflict types, by reducing speed differentials at intersections, and by forcing drivers to decrease speeds as they proceed into and through the intersection. Though roundabout crash records in China are limited, the experiences of other countries can be used to help design roundabouts in China. Understanding the sensitivity of geometric element parameters, along with the crash experience, will assist the designer in optimizing the safety of all vehicle occupants, pedestrians, and bicyclists.

Research indicates that well-designed roundabouts can be safer and more efficient than conventional intersections. A December 2002 report by the Maryland Highway Administration indicates that 15 single-lane roundabouts have greatly improved intersection safety in that State. The analysis shows a 100 percent decrease in the fatal crash rate; a 60 percent decrease in the total crash rate; an 82 percent reduction in the injury crash rate; and a 27 percent reduction in the property damage-only accident rate. In addition, talk about benefits, Crashes are less severe than other intersection crashes; Safer than traditional intersections; cost-effective way to improve intersection safety.

\section{Roundabouts safety analysis}

\subsection{General situation}

Many studies have found that one of the benefits of roundabout installation is the improvement in overall safety performance. Several studies in the U.S., Europe, and Australia have found that roundabouts perform better in terms of safety than other intersection forms. Although the frequency of reported crashes is not always lower at roundabouts, the 
reduced injury rates are usually reported. Safety is better at small and medium capacity roundabouts than at large or multilane roundabouts, While overall crash frequencies have been reduced, the crash reductions are most pronounced for motor vehicles, less pronounced for pedestrians, and equivocal for bicyclists.

The reasons for the increased safety level at roundabouts are:

1. Roundabouts have fewer conflict points in comparison to conventional intersections. The potential for hazardous conflicts, such as right angle and left turn head-on crashes is eliminated with roundabout use. Single-lane approach roundabouts produce greater safety benefits than multilane approaches because of fewer potential conflicts between road users, and because pedestrian crossing distances are short.

2. Low absolute speeds associated with roundabouts allow drivers more time to react to potential conflicts, also helping to improve the safety performance of roundabouts.

3. Since most road users travel at similar speeds through roundabouts, i.e., have low relative speeds, crash severity can be reduced compared to some traditionally controlled intersections.

\subsection{Conflicts analysis}

The frequency of crashes at an intersection is related to the number of conflict points at an intersection, as well as the magnitude of conflicting flows at each conflict point. A conflict point is a location where the paths of two motor vehicles, or a vehicle and a bicycle or pedestrian queue, diverge, merge, or cross each other.

The following sections present a variety of conflicts among vehicles, bicycles, and pedestrians. Both legal conflicts (queuing at an intersection, merging into a traffic stream) and conflicts prohibited by law or by traffic control devices (failure to yield to pedestrians, running a stop sign) have been included for completeness. Even though traffic control devices can significantly reduce many conflicts, they can not eliminate them entirely due to violations of those devices. Many of the most serious crashes are caused by such violations.

\subsection{Crash Statistics}

This section summarizes the overall safety performance of roundabouts in various countries, and then examines the detailed collision types experienced in France and Queensland, Australia.

\subsubsection{Comparisons to previous intersection treatment}

Table1 shows the crash frequencies (average annual crashes per roundabout) experienced at eleven intersections in the U.S. that were converted to roundabouts. As the table shows, both types of roundabouts showed a reduction in both injury and property-damage crashes after installation of a roundabout. It should be noted that due to the small size of the data sample, the only result that is statistically significant is the injury crash reduction for small and moderate roundabouts.

Table1 Average annual crash frequencies at 11 U.S. intersections converted to roundabouts.

\begin{tabular}{lllll}
\hline Type of Roundabout & Sites & $\begin{array}{l}\text { Before Roundabout } \\
\text { Total Inj1. PDO2 }\end{array}$ & $\begin{array}{l}\text { Roundabout } \\
\text { Total Inj. PDO }\end{array}$ & $\begin{array}{l}\text { Percent Change3 } \\
\text { Total Inj. PDO }\end{array}$ \\
\hline Small/Moderate1 & 8 & 4.82 .02 .4 & 2.40 .51 .6 & $-51 \% 73 \%-32 \%$ \\
Large2 & 3 & 21.55 .815 .7 & 15.34 .011 .3 & $-29 \%-31 \%-10 \%$ \\
Total & 11 & 9.33 .06 .0 & 5.91 .54 .2 & $-37 \%-51 \%-29 \%$ \\
\hline
\end{tabular}

1. Inj. = Injury crashes

2. PDO = Property Damage Only crashes

3. Only injury crash reductions for small/moderate roundabouts were statistically significant.

Compared to results from Australia, France, and the United Kingdom, these crash frequencies are quite high. Annual crash frequencies in France, Australia, and United Kingdom of 0.15, 0.6, and 3.31 injury crashes per 
roundabout, respectively, have been reported. The reader should note that the UK has many high-volume, multilane roundabouts.

In spite of the higher frequencies, injury crash rates, which account for traffic volume exposure, are significantly lower at U.S. roundabout sites. In a recent study of eight single-lane roundabouts in Maryland and Florida, the injury crash rate was found to be 0.08 crashes per million entering vehicles. By comparison, the injury crash rate was reported to be 0.045 crashes per million entering vehicles in France and 0.275 crashes per million entering vehicles in the United Kingdom

Experiences in the United States show a reduction in crashes after building a roundabout of about 37 percent for all crashes and 51 percent for injury crashes. These values correspond with international studies with much larger sample sizes, as shown in table2.

table2 Mean crash reductions in various countries.

\begin{tabular}{lll}
\hline \multirow{2}{*}{ Country } & \multicolumn{2}{c}{ Mean Reduction (\%) } \\
& All Crashes & Injury Crashes \\
\hline Australia & $41 \sim 61 \%$ & $45 \sim 87 \%$ \\
France & & $57 \sim 78 \%$ \\
Germany & $36 \%$ & \\
Netherlands & $47 \%$ & \\
United Kingdom & & $25 \sim 39 \%$ \\
United States & $37 \%$ & $51 \%$ \\
\hline
\end{tabular}

The findings of these studies show that injury crashes are reduced more dramatically than crashes involving property damage only. This again is in part due to the configuration of roundabouts, which eliminates severe crashes such as left turn, head-on, and right angle collisions. Most of these studies also show that crash reduction in rural areas is much higher than in urban areas.

Note that the geometry of many studied sites may not necessarily conform to good roundabout design. Improved design principles, such as an emphasis on achieving consistent speeds, may result in better safety performance. It should also be noted that these crash reductions are generally for sites where roundabouts were selected to replace problem intersections. Therefore, they do not necessarily represent a universal safety comparison with all other intersection types.

Collisions at roundabouts tend to be less severe than at conventional intersections. Most crashes reported at roundabouts are a result of drivers failing to yield on entry, referred to as entering-circulating crashes. In addition, rear-end collisions and single vehicle crashes have been reported in many studies. Table 3 shows the percentage of the three main crash types reported in different countries.

Table3 Reported proportions of major crash types at roundabouts.

\begin{tabular}{llllll}
\hline \multirow{2}{*}{ Country } & \multirow{2}{*}{ Crash Description } & Type of Roundabout & \multicolumn{3}{c}{ Type of Crash1 } \\
& & & Entering circulating & Rear-end & Single Vehicle \\
\hline Australia & All crashes & Single and multilane & $51 \%$ & $22 \%$ & $18 \%$ \\
France & Injury crashes & Single and multilane & $37 \%$ & $13 \%$ & $28 \%$ \\
Germany & All crashes & Single lane & $30 \%$ & $28 \%$ & $17 \%$ \\
Switzerland & All crashes & Single and multilane & $46 \%$ & $13 \%$ & $35 \%$ \\
United & Injury crashes & Single and multilane & $20-71 \%$ & $7-25 \%$ & $8-30 \%$ \\
Kingdom & & & & & \\
\hline
\end{tabular}

1. Percentages do not necessarily sum to $100 \%$ because only three major crash categories are shown. 


\subsubsection{Collision types}

It is instructive for designers to examine details of collision types and location at roundabouts. Statistics are available for roundabouts designed according to local practices in France, Queensland (Australia), and the United Kingdom. It should be noted that the reported frequencies are to some extent related to the specific design standards and reporting processes used in these countries.

Table4 presents a summary of the percentage of crashes by collision type. For comparison purposes, data from France, Queensland (Australia) and the United Kingdom have been superimposed onto the same classification system.

Table4 Comparison of collision types at roundabouts.

\begin{tabular}{llll}
\hline Collision Type & France & Queensland (Australia) & United Kingdom1 \\
\hline 1. Failure to yield at entry (entering-circulating) & $36.6 \%$ & $50.8 \%$ & $71.1 \%$ \\
2. Single-vehicle run off the circulatory roadway & $16.3 \%$ & $10.4 \%$ & $8.2 \%$ \\
3. Single vehicle loss of control at entry & $11.4 \%$ & $5.2 \%$ & \\
4. Rear-end at entry & $7.4 \%$ & $16.9 \%$ & $7.0 \%$ \\
5. Circulating-exiting & $5.9 \%$ & $6.5 \%$ & $3.5 \%$ \\
6. Pedestrian on crosswalk & $5.9 \%$ & & 2 \\
7. Single vehicle loss of control at exit & $2.5 \%$ & $2.6 \%$ & \\
8. Exiting-entering & $2.5 \%$ & & \\
9. Rear-end in circulatory roadway & $0.5 \%$ & $1.2 \%$ & \\
10. Rear-end at exit & $1.0 \%$ & $0.2 \%$ & \\
11. Passing a bicycle at entry & $1.0 \%$ & & \\
12. Passing a bicycle at exit & $1.0 \%$ & & 4 \\
13. Weaving in circulatory roadway & $2.5 \%$ & $2.0 \%$ & $10.2 \%$ \\
14. Wrong direction in circulatory roadway & $1.0 \%$ & & \\
15. Pedestrian on circulatory roadway & $3.5 \%$ & & \\
16. Pedestrian at approach outside crosswalk & $1.0 \%$ & & \\
Other collision types & & $2.4 \%$ & \\
Other sideswipe crashes & & $1.6 \%$ & \\
\hline
\end{tabular}

1. Data are for "small" roundabouts (curbed central islands $>4 \mathrm{~m}[13 \mathrm{ft}]$ diameter, relatively large ratio of inscribed circle diameter to central island size)

Three of the predominant types of collision are:

(1) failures to yield at entry to circulating vehicles

(2) single vehicle run-off the circulatory roadway

(3) single vehicle run-into the central island. A more recent crash study confirmed a high proportion of single vehicle crashes: 49 percent in rural areas, versus 21 percent in urban areas.

To reduce the severity of single vehicle crashes, special attention should be accorded to improving visibility and avoiding or removing any hard obstacles on the central island and splitter islands in both urban and rural environments. A French study identified a number of major obstacles that caused fatalities and injuries: trees, guardrail, concrete barriers, fences, walls, piers, sign or light poles, landscaping pots or hard decorative objects, and steep cross-slopes on the central island.

\section{Conclusion}

As with crash analyses, conflict analyses are more than the simple enumeration of the number of conflicts. A conflict analysis should account for the following factors: Existence of conflict point; Exposure, measured by the product of the two conflicting stream volumes at a given conflict point; Severity, based on the relative velocities of the 
conflicting streams (speed and angle); and Vulnerability, based on the ability for a member of each conflicting stream to survive a crash.

For the design of a new roundabout, safety can be optimized not only by relying on recorded past performance of roundabouts in general, but primarily by applying all design knowledge proven to impact safety. For optimum roundabout safety and operational performance the following should be noted:

(1). Minimizing the number of potential conflicts at any geometric feature should reduce the multiple vehicle crash rate and severity.

(2) Minimizing the potential relative speed between two vehicles at the point of conflict will minimize the multiple vehicle crash rate and severity (it may also optimize capacity). To reduce the potential relative speed between vehicles, either the absolute speeds of both vehicles need to be reduced or the angle between the vehicle paths needs to be reduced.

(3) Limiting the maximum change in speed between successive horizontal geometric elements will minimize the single vehicle crash rate and severity.

\section{References}

[1] “U.S. Department of Transportation contents "chapter5-safety, 2003.

[2] SS Nambisan, V Parimi, A Comparative Evaluation of the Safety Performance of Roundabouts and Traditional Intersection Controls, Institute of Transportation Engineers, pp. 18-25, 2007.

[3] R Elvik, Effects on Road Safety of Converting Intersections to Roundabouts: Review of Evidence from Non-U.S. Studies, Transportation Research Record Journal of the Transportation Research Board,2003. 